\title{
Ein Satz aus der Theorie der unendlichen Reihen.
}

\author{
Von A. Tauber in Wien.
}

Ist $\sum_{I}^{\infty} a_{\nu}$ irgend eine convergente Reihe, so müssen die Größen $a_{\nu}$ den folgenden 2 Bedingungen Genüge leisten: Erstens muss (nach einem A bel'schen Satze), wenn $p$ eine positive Größse kleiner als 1 bedeutet, die Function $\sum_{1}^{\infty} a_{\nu} \rho^{\nu}$ von $\rho$ für $\lim \rho=1$ einen Grenzwert besitzen und zweitens muss (nach einem Satz von Krone(ker) ${ }^{1}$ )

$$
\lim _{n=\infty} \frac{1}{n} \sum_{\nu=1}^{n} v a_{\nu}=0
$$

sein. Umgekehrt aber darf weder daraus, dass $\lim _{\varrho=1} \sum_{1}^{\infty} a_{\nu} \rho^{\nu}$ existiert, noch daraus, dass $\lim _{n=\infty} \frac{1}{n} \sum_{1}^{n} v a_{\nu}=0$ ist, auf die Convergenz der Reihe $\sum_{1}^{\infty} a_{\nu}$ geschlossen werden. Wenn dagegen beide Bedingungen zugleich erfüllt sind, so convergiert $\sum_{1}^{\infty} a_{\gamma}$, sodass also der Satz zu beweisen ist:

Damit irgend eine Reihe $\sum_{1}^{\infty} a_{\nu}$ convergiert, ist erforder(A) lich und hinreichend, dass gleichzeitig $\lim _{\varrho=1} \sum_{1}^{\infty} a_{v} \rho^{v}$ existiert und dass $\lim _{n=\infty} \frac{1}{n} \sum_{1}^{n} v a_{\nu}=0$ ist.

Wir beweisen den Satz $(A)$ zunächst für den Fall, dass $\lim v a_{v}=0$ ist, in der folgenden Form:

1) Kronecker, Comptes rendus, T. 103, pag. 980.

Monatsh. f. Mathematik u. Physik. VIII. Jahrg. 
Sind $b_{1}, b_{2}, \ldots$ irgendwelche die Bedingung $\lim v b_{v}=0$ (B) erfüllende Größen, und ist $\lim _{\varrho=1} \sum_{1}^{\infty} b_{\nu} \rho^{\nu}{ }_{\infty}$ gleich einer bestimmten endlichen Größe $A$, so convergiert $\sum_{1}^{\infty} b$, und hat $A$ zur Summe.

Setzen wir, um dies zu zeigen, $\psi(\rho)=\sum_{1}^{\infty} b_{\nu} \rho^{\nu}$, ferner $\left|b_{v}\right|=\beta_{v}$ und nennen $\tau_{n}$ die obere Grenze der Größen

$$
(n+1) \beta_{n+1},(n+2) \beta_{n+2}, \ldots,
$$

so ist

$$
\psi\left(1-\frac{1}{n}\right)=\sum_{\nu=1}^{n} b_{\nu}\left(1-\frac{1}{n}\right)^{\nu}+\sum_{\nu=n+1}^{\infty} b_{\nu}\left(1-\frac{1}{n}\right)^{\nu}
$$

und

$$
\begin{aligned}
\left|\sum_{\nu=n+1}^{\infty} b_{\nu}\left(1-\frac{1}{n}\right)^{\nu}\right| & \leqq \sum_{\nu=n+1}^{\infty} \beta_{\nu}\left(1-\frac{1}{n}\right)^{\nu}<\sum_{\nu=n+1}^{\infty} \frac{\nu}{n} \beta_{\nu}\left(1-\frac{1}{n}\right)^{\nu} \\
& <\frac{\tau_{n}}{n} \sum_{\nu=n+1}^{\infty}\left(1-\frac{1}{n}\right)^{\nu}<\tau_{n}\left(1-\frac{1}{n}\right)^{n+1} \\
& <\tau_{n},
\end{aligned}
$$

daher erbält man, wenn $\vartheta$ eine Größe ist, deren Betrag kleiner als 1 ist,

$$
\sum_{\nu=1}^{n} b_{v}-\psi\left(1-\frac{1}{n}\right)=\sum_{\nu=1}^{n} b_{v}\left[1-\left(1-\frac{1}{n}\right)^{\nu}\right]+\vartheta \tau_{n} .
$$

Offenbar ist

$$
0<1-\left(1-\frac{1}{n}\right)^{v} \leq \frac{\nu}{n} \quad \text { für } \nu \geq 1
$$

und deshalb

$$
\begin{gathered}
\left|\sum_{\nu=1}^{n} b_{\nu}\left[1-\left(1-\frac{1}{n}\right)^{\nu}\right]\right|<\sum_{\nu=1}^{n} \frac{\nu \beta_{\nu}}{n} \\
\left|\sum_{1}^{n} b_{\nu}-\psi\left(1-\frac{1}{n}\right)^{\nu}\right|<\frac{1}{n} \sum_{1}^{n} \nu \beta_{\nu}+\Sigma_{n} .
\end{gathered}
$$


Für $\lim _{\nu=\infty} \nu \beta_{\nu}=0$ wird aber sowohl $\tau_{n}$ als $\frac{1}{n} \sum_{1}^{n} \nu \beta_{v}$ mit wachsendem $n$ beliebig klein, somit resultiert die zu beweisende Gleichung

$$
\lim _{n=\infty}\left[\bigcup\left(1-\frac{1}{n}\right)-\sum_{1}^{n} b_{\nu}\right]=0 .
$$

Neben dem Satz $(B)$ besteht auch noch der folgende: Sind $b_{1}, b_{2}, \ldots$ irgendwelche die Bedingung $\lim _{\nu=\infty} b_{v}=0$ erfüllende Größen, and bezeichnet man $\Sigma b_{\nu} \rho^{\nu}$ mit $\psi(\rho)$, so ist

$$
\lim _{\varrho=1}\left[(1-\rho) \varphi^{\prime}(\rho)\right]=0 \text {. }
$$

Setzt man nämlich in

$$
\begin{gathered}
(1-\rho) \varphi^{\prime}(\rho)=(1-\rho) \sum_{1}^{\infty} \nu b_{\nu} \rho^{\nu-1} \\
\rho=1-\frac{1}{n+\Theta} \quad(0 \leq \Theta<1),
\end{gathered}
$$

so ist

$$
\begin{aligned}
\left|(1-\rho) \sum_{1}^{n} \nu b_{\nu} \rho^{\nu-1}\right| & \leq(1-\rho) \sum_{1}^{n} \nu \beta_{\nu} \rho^{\nu-1} \mid<\frac{1}{n} \sum_{1}^{n} \nu \beta_{\nu} \\
(1-\rho) \sum_{n+1}^{\infty} \nu b_{\nu} \rho^{\nu-1} \mid & \leq(1-\rho) \sum_{n+1}^{\infty} \nu \beta_{\nu} \rho^{\nu-1}<(1-\rho) \tau_{n} \sum_{n+1}^{\infty} \rho^{\nu-1} \\
& <\tau_{n} \rho^{n},
\end{aligned}
$$

wenn wieder $\tau_{n}$ die obige Bedeutung hat; somit nähert sich die rechte Seite in (3) für $\lim p=1$ der Null.

Seien jetzt irgendwelche Größen $a_{1}, a_{2}, \ldots, a_{v}, \ldots$ vorgelegt, und mit Hilfe derselben die Größen $b_{\nu}$ definiert durch

$$
\begin{aligned}
& b_{\nu}=\frac{a_{1}+2 a_{2}+\cdots+\nu a_{\nu}}{\nu(\nu+1)}, \quad \nu \geq 1 . \\
& b_{0}=0 .
\end{aligned}
$$

Aus diesen Gleichungen folgt

$$
\begin{aligned}
a_{v} & =(\nu+1) b_{v}-(\nu-1) b_{\nu-1} \quad \nu \geq 1 \\
\sum_{1}^{n} a_{v} & =\sum_{1}^{n}(\nu+1) b_{v}-\sum_{1}^{n}(\nu-1) b_{\nu-1} \\
& =(n+1) b_{n}+\sum_{1}^{n-1}(v-1-1) b_{v}-\sum_{1}^{n}(\nu-1) b_{\nu-1},
\end{aligned}
$$


und daher ist

$$
\sum_{1}^{n} a_{\nu}=(n+1) b_{n}+\sum_{1}^{n-1} b_{\nu} .
$$

Zwischen den beiden Functionen

$$
\begin{aligned}
& \varphi(\rho)=\sum_{1}^{\infty} a_{\nu} \rho^{\nu} \\
& \psi(p)=\sum_{1}^{\infty} b_{\nu} \rho^{\nu}
\end{aligned}
$$

besteht wegen der Gleichungen

$$
\begin{aligned}
\varphi(\rho)=\sum_{1}^{\infty} a_{\nu} \rho^{\nu} & =\sum_{1}^{\infty}\left[(\nu+1) b_{\nu}-(\nu-1) b_{\nu-1}\right] \rho^{\nu} \\
& =\sum_{1}^{\infty}(\nu+1) b_{\nu} \rho^{\nu}-\sum_{1}^{\infty} \nu b_{\nu} \rho^{\nu+1} \\
& =\sum_{1}^{\infty} b_{\nu} \rho^{\nu}+\sum_{1}^{\infty} \nu b_{\nu} \rho^{\nu}(1-\rho)
\end{aligned}
$$

der Zusammenhang

$$
\varphi(p)-\psi(p)=p(1-p) \psi^{\prime}(p),
$$

und der Satz $(A)$ beweist sich nun folgendermaßen: Erfüllen die Größen $a_{\nu}$ die Bedingung

$$
\lim _{n=\infty} \frac{1}{n} \sum_{1}^{n} v a_{\nu}=0
$$

so besitzen die durch (4) definierten Größen $b_{\nu}$ die Eigenschaft $\lim _{\nu=\infty}(\nu+1) b_{\nu}=0$ oder

$$
\lim _{\nu=\infty} \nu b_{v}=0
$$

ist aber $\lim \nu b_{\nu}=0$, so folgt aus (2), dass

$$
\left.\lim _{\varrho=1}[(1-\rho)\rfloor^{\prime}(\rho)\right]=0,
$$

d. h. dass nach (7)

$$
\lim _{\varrho=1}[భ(p)-\psi(p)]=0
$$


ist. Weil ferner der Voraussetzung nach $\lim \varphi(\rho)$ eine endliche bestimmte Größe $A$ sein sollte, so muss nach $(8)$ auch $\lim \psi(\rho)=A$ sein, und demnach gemäß dem Satze $(B)$ die Reihe $\Sigma b_{y}$ convergieren und $A$ zur Summe haben. Und endlich folgt aus der Convergenz von $\sum b_{v}$, und $\lim _{v=\infty} \nu b_{v}=0$, dass nach (6) auch $\sum a_{v}$ convergiert und $A$ zur Summe hat. 